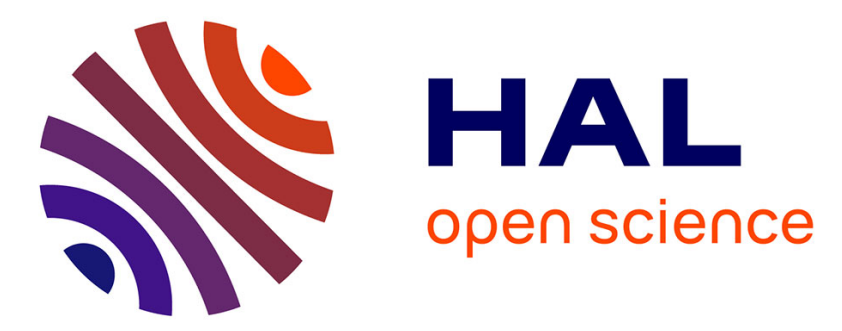

\title{
Judging where a ball will go: the case of curved free kicks in football
}

Cathy Craig, Eric Berton, Guillaume Rao, Laure Fernandez, Reinoud J. Bootsma

\section{> To cite this version:}

Cathy Craig, Eric Berton, Guillaume Rao, Laure Fernandez, Reinoud J. Bootsma. Judging where a ball will go: the case of curved free kicks in football. The Science of Nature Naturwissenschaften, 2006, 93 (2), pp.97-101. 10.1007/s00114-005-0071-0 . hal-01635484

\section{HAL Id: hal-01635484 \\ https://hal-amu.archives-ouvertes.fr/hal-01635484}

Submitted on 15 Nov 2017

HAL is a multi-disciplinary open access archive for the deposit and dissemination of scientific research documents, whether they are published or not. The documents may come from teaching and research institutions in France or abroad, or from public or private research centers.
L'archive ouverte pluridisciplinaire HAL, est destinée au dépôt et à la diffusion de documents scientifiques de niveau recherche, publiés ou non, émanant des établissements d'enseignement et de recherche français ou étrangers, des laboratoires publics ou privés. 


\section{Judging where a ball will go: the case of curved free kicks in football}

Received: 29 June 2005 / Accepted: 8 November 2005 / Published online: 1 February 2006

(C) Springer-Verlag 2006

\begin{abstract}
This study examined whether adding spin to a ball in the free kick situation in football affects a professional footballer's perception of the ball's future arrival position. Using a virtual reality set-up, participants observed the flight paths of aerodynamically realistic free kicks with $( \pm 600 \mathrm{rpm})$ and without sidespin. With the viewpoint being fixed in the centre of the goal, participants had to judge whether the ball would have ended up in the goal or not. Results show that trajectories influenced by the Magnus force caused by sidespin gave rise to a significant shift in the percentage of goal responses. The resulting acceleration that causes the ball to continually change its heading direction as the trajectory unfolds does not seem to be taken into account by the participants when making goal judgments. We conclude that the visual system is not attuned to such accelerated motion, which may explain why goalkeepers appear to misjudge the future arrival point of such curved free kicks.
\end{abstract}

\section{Introduction}

Numerous studies have highlighted the discrepancy that exists between how falling objects behave in the physical world, as described by Newtonian physics, and how we perceive these events (Shanon 1976; Zago 2005). One

Electronic Supplementary Material Supplementary material is available for this article at http://dx.doi.org/10.1007/s00114-0050071-0.

C. M. Craig $\cdot$ E. Berton $\cdot$ G. Rao $\cdot$ L. Fernandez $\cdot$ R. J. Bootsma Movement and Perception, University of the Mediterranean, Marseille, France

C. M. Craig $(\bowtie)$

School of Psychology,

Queen's University Belfast,

Belfast, Northern Ireland

e-mail: cathy.craig@qub.ac.uk

Tel.: +44-289097-5482

Fax: +44-289066-4144 classic example is the rising fastball illusion in baseball where players claim that the ball rises the moment it crosses the plate. Such a phenomenon could only physically exist if the ball travelling at $40 \mathrm{~m} / \mathrm{s}$ was spinning (backwards) at over 3,600 rpm, so as to create a Magnus force that would counteract the effects of gravity. As maximum spin rates achieved by top-level pitchers do not exceed 2,300 rpm, such an explanation is untenable (Bahill and Karnavas 1993). Instead, a discrepancy must exist between how the ball physically moves as it approaches the plate and where the batter expects the ball to go McBeath (1990). Why would batters be unable to correctly predict where a rising fastball will go, given their remarkable level of skill in hitting and intercepting balls in a wide variety of situations? Are they led to make errors in estimating ball speed (Bahill and Karnavas 1993; McBeath 1990) or could the perceptual effect be partially due to the pattern of spin that causes the ball to deviate from a standard (quasi-parabolic) trajectory?

To investigate the effects of spin on perception, we turned to the game of football where the Magnus effect is exploited to produce curved ball trajectories. In modern day football, free kicks are becoming important goal-scoring opportunities (Grant et al. 1999), with specialist free kick takers often opting to impart sidespin on the ball so as to curve the ball into the goal. What are the implications of these curved free kicks for goalkeepers? Is the future heading direction of a ball following a curved flight path more difficult to perceive? We suggest that the continuous non-linear change in the direction of the ball's flight, caused by the Magnus force, will affect the goalkeeper's ability to accurately perceive the ball's future arrival point.

To better understand the implications of the Magnus force for the visual system, one must consider the physical consequences of such a force on a ball's trajectory. The Magnus force, created by a ball spinning around an axis, gives rise to an acceleration that is perpendicular to the direction of the current orientation of the velocity vector and is contained within the plane of rotation (Fig. 1a, S1). Although studies that have examined the perceptual effects of this type of acceleration are few and far between, the (in) sensitivity of the human visual system to other forms of 
A

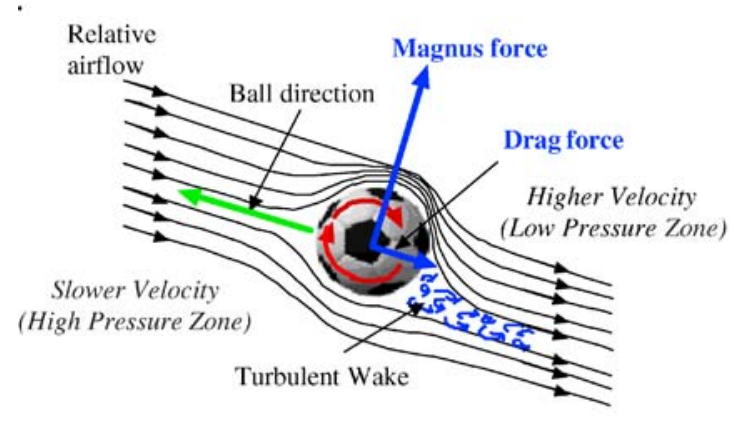

Fig. 1 Panel a shows a schematic representation of the airflow around a rotating football during flight. The red arrows represent the direction of spin. Given the direction of ball travel (green arrow), air flowing clockwise around the ball will move faster than the air moving counter-clockwise around the ball causing a pressure

accelerated motion has been studied quite extensively (Babler and Dannemiller 1993; Todd 1981). Werkhoven et al. (1992) have shown that acceleration as such is not detected by the visual system but may be inferred through a two-step mechanism where initial and final velocities are compared. Recently, Brouwer et al. (2002) demonstrated that a $25 \%$ change in velocity, under optimal circumstances, is needed for acceleration to be detected at all.

It therefore seems unlikely that the visual system is able to provide the accurate measure of acceleration that would be needed to predict the spatio-temporal trajectory of an accelerating object (Port et al. 1997). Indeed, the unfolding of movement during interceptive actions is better characterised by a reliance on prospective information carried in the perceptual flow than on computation-based extrapolations of the current situation (Bootsma and Van Wieringen 1990; Lee et al. 1983; McLeod and Dienes 1996). While numerous studies have dealt with the timing of interceptive actions (Bootsma and Van Wieringen 1990; Lee et al. 2001; Montagne et al. 1999), few have addressed the issue of where a moving object is going (Peper et al. 1994; Regan 1997). In the case of football, the size of the goalmouth $(7.32 \mathrm{~m})$ suggests that a goalkeeper needs to anticipate in what direction the ball is going before engaging in action. To examine whether the lateral deflection of a ball's trajectory, caused by the Magnus force created by side spin, has an effect on a professional footballer's perception of where a ball is going, we asked our professional football participants to judge whether a range of (simulated) free kicks would end up in the goal or not. For each of eight different arrival positions (Fig. 2), the ball could follow one of three different trajectories; non-deflected (zero spin), deflected by a 600-rpm sidespin in a counter-clockwise direction or deflected by a 600 -rpm sidespin in a clockwise direction (Fig. 3, S1). Although many other factors such as the type of surface or air density can also alter the char-
B

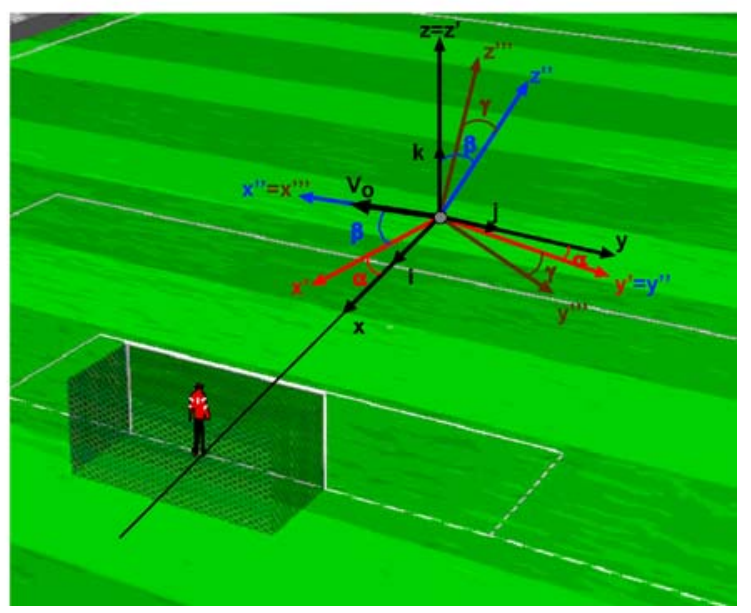

differential which gives rise to the Magnus force. Note the direction of the Magnus force, orthogonal to the direction of motion, causing a lateral deviation in the ball's trajectory. Panel b shows the reference coordinates that were used in the mathematical modelling (S1) of the simulated free kicks

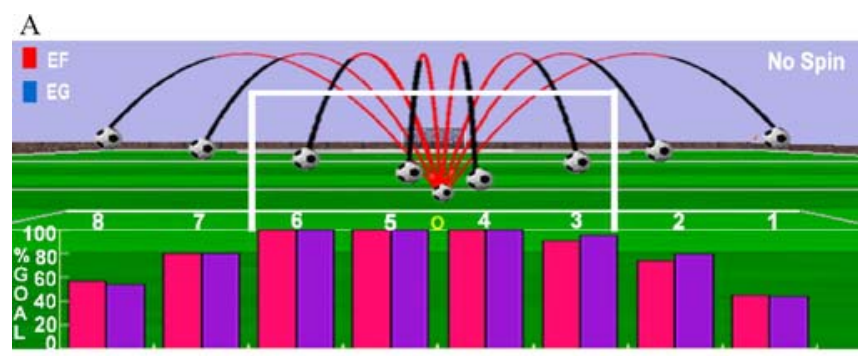

B

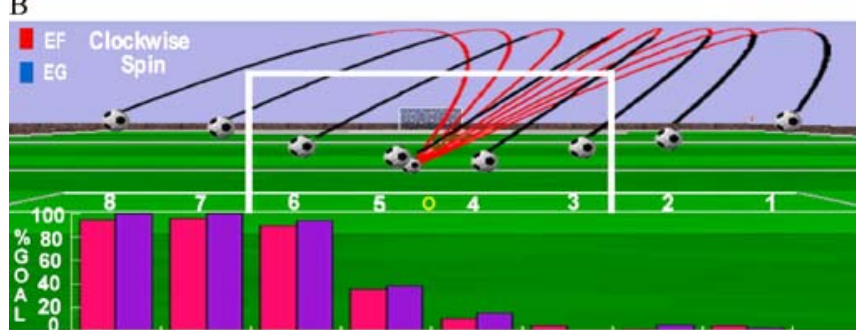

$\mathrm{C}$

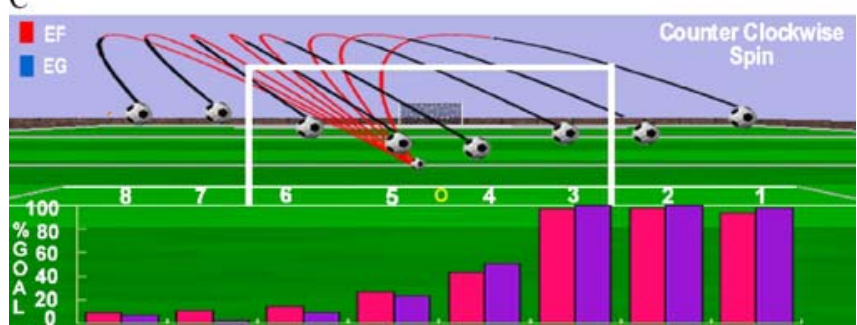

Fig. 2 Panels a, b and $\mathbf{c}$ show the mean percentage of goal responses for each of the two different groups - expert field players $(E F$, red $)$, expert goalkeepers (EG, purple) - for the eight arrival points (1-8 on the $x$-axis). Panel a shows the results for the no spin condition (NS), b the clockwise spin condition (CS) and $\mathbf{c}$ the counter-clockwise spin condition (CCS). $O$ corresponds to the point of observation. These mean results are for the $10.0-\mathrm{m}$ cut-off condition only with the black lines indicating the section that the participants did not see 
Fig. 3 Evolution of ball trajectories over time for two different arrival positions ( 3 and 5 , left and right columns, respectively). Panels a and b present superimposed images captured from the virtual reality programme showing the progression of the ball every $160 \mathrm{~ms}$ for each of the three different trajectories (CS clockwise spin, $N S$ no spin, CCS counter-clockwise spin). Panel a illustrates what the observer saw when judging whether the ball would land in the goalmouth or not, whilst panel b shows the same trajectories from the free kick taker's perspective. Panel c shows a bird's eye view of the trajectories, highlighting how sidespin affects the lateral position of the ball. The dotted lines represent the trajectory tangents at 30,20 and $10 \mathrm{~m}$ from the goal line. The intersection point along the goal line indicates the arrival position for the ball had the effects of the Magnus Force stopped at that precise moment in time (linear extrapolation). Panel d shows how the arrival position, as determined by linear extrapolation of the ball's trajectory tangent at each time step following the logic presented in c, varies as the ball approaches the goal line (see S1 for details)
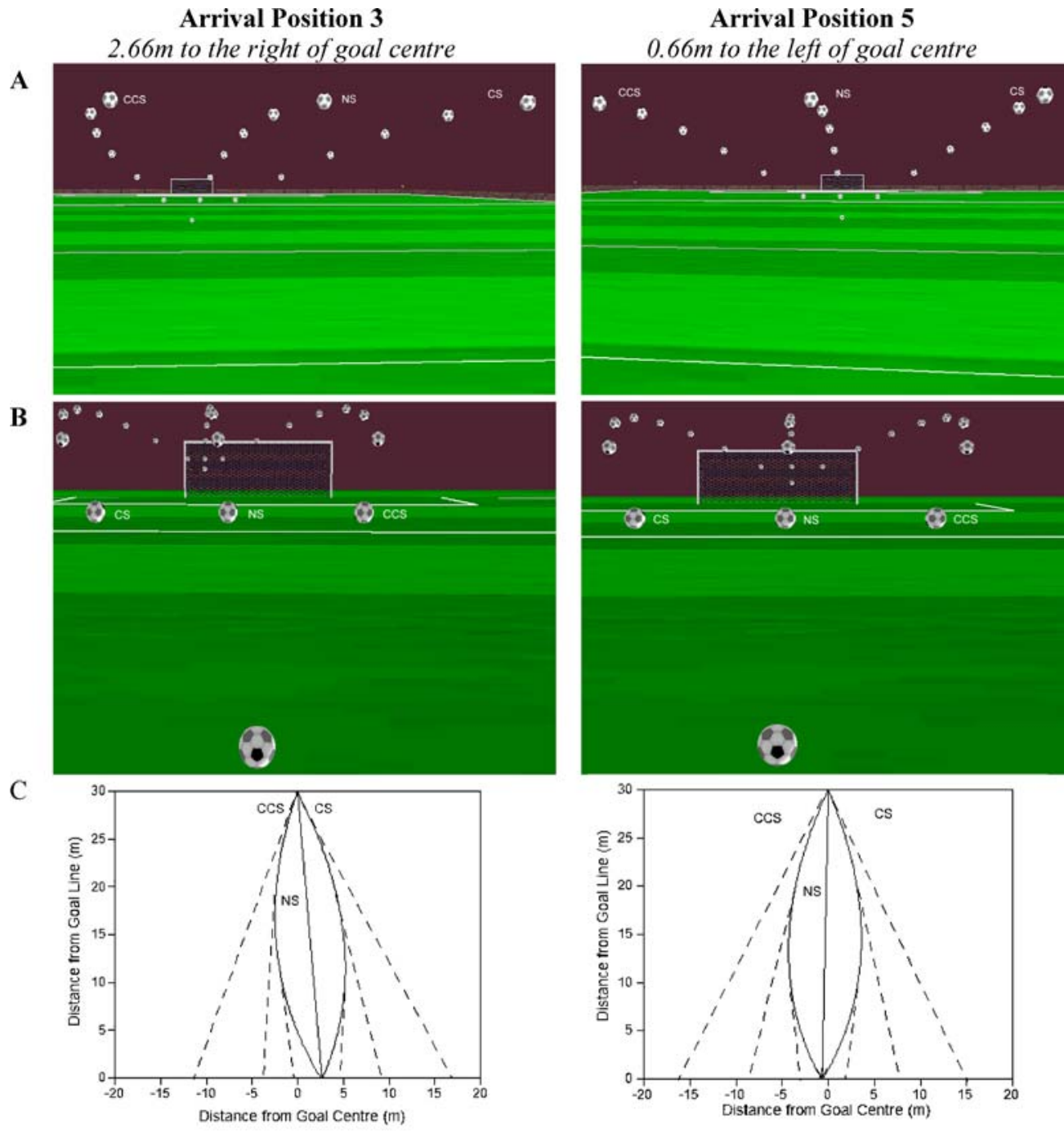

D

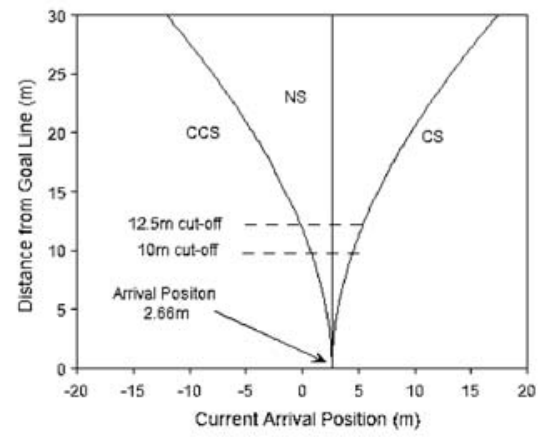

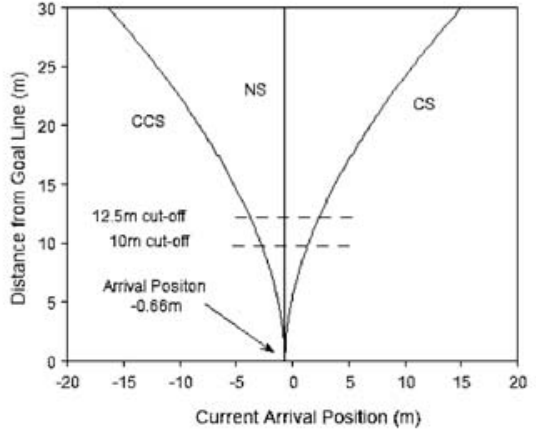

acteristics of a projectile's flight path (Carré et al. 2002; de Mestre 1990), this study focused uniquely on the effects of realistic spin rates in free kick situations on judgements of the future arrival position of the ball.

\section{Materials and methods}

A theoretical model incorporating aerodynamic MagnusRobins lift and drag forces was developed (Fig. 1, S1). With all trajectories starting from the same initial position $(30 \mathrm{~m}$ from the goal), eight no-spin trajectories were generated. The trajectories differed only in the direction of the initial velocity vector $(36.0 \mathrm{~m} / \mathrm{s}$ constant magnitude $)$ so as to reach eight designated arrival positions $(0.66,2.66,4.66,6.66 \mathrm{~m}$ to the left and right of centre of the goal, Fig. 2). Again, adapting only the direction of the initial velocity vector, trajectories with the ball spinning clockwise $(-600 \mathrm{rpm})$ and counter-clockwise $(+600 \mathrm{rpm})$ around the vertical axis were calculated so that they arrived at exactly the same positions. Each arrival position had three different trajectory forms, giving rise to a total of 24 free kicks. Total 
flight times varied from 1.35 to $1.41 \mathrm{~s}$ depending on the final arrival position.

The data specifying the trajectories were used to animate, in real-time, a black and white textured sphere corresponding to the colour and size of a real football in a virtual environment. Use of a virtual reality setting not only guaranteed exact reproduction of ball trajectories, but also allowed for the elimination of other factors, such as occlusion of the ball by defensive players and the preparatory movements of the kicker (Abernethy 1991; Williams and Griffiths 2002). The virtual football stadium was constructed so that the pitch, goalposts, line markings, etc., all conformed to FIFA regulations (Fig. 2; S2).

The simulated free kicks were presented to two different groups of observers: 11 expert footballers (attackers, midfielders and defenders) and nine expert goalkeepers. The players, drawn from the professional footballing squads at AC Milan (Italy), Olympique de Marseille (France), Bayer Leverkusen and Schalke 04 (both Germany), had an average age of 25.1 years (range 19 to 34 ).

Observers viewed the virtual football stadium through a head-mounted display unit (Cybermind Hi-Res900, field of view $31.5^{\circ}$ each eye). An integrated head tracking system (Flock of Birds electromagnetic sensor, Ascension Technologies) linked the viewpoint in the virtual world (delay $<30 \mathrm{~ms}$ ) with rotations of the head, whilst maintaining the location of observation at a fixed point $(1.8 \mathrm{~m}$ above the ground) in the centre of the goal.

During an initial exploratory phase, participants were asked to turn their heads right, left, up and down, so as to get their bearings in the goalmouth (S2). The training period that followed allowed participants to become familiar with the task of judging, by pressing the appropriate mouse button, whether a ball that disappeared from the scene at a distance of either 10.0 or $12.5 \mathrm{~m}$ from the goal line, would have ended up in the goal or not. No goals corresponded to balls going wide of the goalposts. Ball height was controlled so that none of the free kicks went over the crossbar. Observers, presented with 32 (two display cut-off distances, eight arrival positions, each presented twice) no-spin free kicks, were given performance feedback (S3).

Response feedback was no longer provided for the main experimental session (S4) of 480 trials (three trajectory types, eight arrival positions, two display cut-off distances repeated ten times). An experimental session lasted on average $1 \mathrm{~h}$ with regular pauses being given.

\section{Results}

As expected, the percentage of "goal" responses varied as a function of the arrival position $\left[F_{(7,126)}=287.79, p<0.001\right]$. For the no-spin trajectories (NS), balls arriving at positions $3,4,5$ and 6 were consistently judged as entering the goal (Fig. 2a). When the ball was going wide, the percent judged goal decreased, from $79.8 \%$ for positions 2 and 7 ( $1-\mathrm{m}$ wide) to $47.5 \%$ for positions 1 and 8 ( $3-\mathrm{m}$ wide). This symmetrical pattern of goal responses observed for the NS trajectories was profoundly changed for the trajectories with spin $\left[F_{(14,252)}=217.82, p<0.001\right]$. When the ball was spinning clockwise (CS), the resulting trajectories, from the point of view of the goalkeeper, unfolded on the right-hand side of the NS trajectory (Fig. 2b). In this case, free kicks arriving at positions 1,2 and 3 were consistently judged as not being goals (on average, 3.0, 3.3 and $3.5 \%$ goal responses, respectively). From positions 4 to 6 the percentage of kicks judged as being a goal steadily increased (from 11.4 to $90.3 \%$ ), reaching a maximum value of $98.8 \%$ for position 7 and declining slightly thereafter $(95.8 \%$ for position 8). For conditions where the ball was spinning counter-clockwise (CCS) with trajectories unfolding on the left-hand side of the standard NS trajectory, the exact opposite pattern was found (Fig. 2c). Positions 8,7 and 6 were now judged as not entering the goal $(5.9,8.1$ and $11.4 \%$, goal responses, respectively). This percentage of goal responses increased from position 5 onwards to reach 97.8 and $97.3 \%$ for positions 3 and 2, respectively, with a slight decrease being observed for position $1(94.2 \%)$. Relative to the no-spin trajectories, trajectories with spin produced clear shifts in the pattern of goal responses, with the direction of the shift being dependent on the direction of the spin.

Whether the ball disappeared at 10.0 or $12.5 \mathrm{~m}$ from the goal did not influence the results obtained, other than through a somewhat lower percentage of goal responses for position 4 in the CS balls ( $42.1 \%$ vs $46.5 \%$ for CCS) and for position 5 in the CCS balls ( $31.6 \%$ vs $36.5 \%$ for CS), as evidenced by the analyses of the interaction between cut-off and arrival position $\left[F_{(7,126)}=2.39, p<0.05\right]$. Finally, whether participants were expert field players or goalkeepers did not have an effect on the percentage of goal responses given [main effect $F_{(1,18)}=0.05 ; p=0.83$, and all interactions involving the factor group: $\mathrm{ps}>0.10]$.

\section{Discussion}

In the present study, we tested the effects of lateral spin on judgements of the future arrival position of the ball. The curving trajectories that resulted from the Magnus force acting in the horizontal plane clearly influenced the pattern of responses obtained. A symmetrical pattern of goal responses was obtained in the NS conditions. This pattern of goal responses shifted to the left (towards position 8) for balls curving under the influence of clockwise sidespin and to the right (towards position 1) for balls curving under the influence of counter-clockwise sidespin. Fig. 3 essentially shows how lateral acceleration caused by the Magnus force affects the ball's heading direction as the trajectory unfolds. Players appear to be using current ball heading direction to make their judgements about whether the free kicks will end up in the goal or not, rather than accurately predicting the effects of lateral acceleration on the ball's future arrival position. As all trajectories emanated from the same initial position, the present data set does not allow us to identify the source(s) of visual information underlying the judgements provided. Further experimentation is necessary to tease out the relevant information variables. 
What is clear, however, is that a curving ball trajectory can induce errors in perceptual judgements of final arrival position in top-level footballers. The results from this study provide strong evidence that the perceptual effects described find their origin in inherent limitations of the human visual system in anticipating the arrival point of an object subjected to an additional accelerative influence resulting from the presence of the Magnus force (see Fig. 3). The depth of experience of our participants does not seem to be able to compensate for these shortcomings in visual perception. The elimination of all potentially confounding contextual information meant that in the present experiment, participants responded only with respect to the information contained in the spatiotemporal pattern of the unfolding ball trajectory. In a more realistic situation, one might expect such expert players' judgements to improve as they can use additional contextual information pertinent to the free kick situation (Abernethy 1991; Williams and Griffiths 2002).

Because at the scale of human performance acceleration due to gravity is an invariant property of the environment, experience with this invariant allows it to be integrated into the processes underlying perception and action (Grealy et al. 2004; McIntyre et al. 2001). However, the acceleration resulting from spin around a given axis is not an invariant property of the environment, as its effects change from one situation to another and even during ball flight (Mehta 1985). With a visual system poorly adapted to accelerated motion, the ever-varying degree and direction of acceleration caused by a spinning ball cannot be integrated into perception and action for purposes of anticipation. The cognitive and behavioural strategies developed by top-level players to avoid being misled certainly merit attention in future studies.

Acknowledgements The authors would like to thank the players and coaches from Olympique de Marseille, AC Milan, Schalke 04 and Bayer Leverkusen who participated in this study; AdidasSalamon Germany who facilitated contact with the clubs, and staff at Milan Lab, Milanello, Italy. The authors also thank Cédric Goulon and Florian Laborde for their assistance in creating the virtual environment.

\section{References}

Abernethy B (1991) Visual search strategies and decision-making in sport. Int J Sport Psychol 22:189-210

Babler TG, Dannemiller JL (1993) Role of image acceleration in judging landing location of free-falling projectiles. J Exp Psychol Hum Percept Perform 19:15-31
Bahill AT, Karnavas WJ (1993) The perceptual illusion of baseball's rising fastball and breaking curveball. J Exp Psychol Hum Percept Perform 19:3-14

Bootsma RJ, Van Wieringen PCW (1990) Timing an attacking forehand drive in table-tennis. J Exp Psychol Hum Percept Perform 16:21-29

Bray D, Kerwin DG (2003) Modelling the flight of a soccer ball in a direct free kick. J Sport Sci 21:75-85

Brouwer AM, Brenner E, Smeets BJ (2002) Perception of acceleration with short presentation times: can acceleration be used in interception? Percept Psychophys 64:1160-1168

Carré MJ, Asai T, Akatsuka T, Haake SJ (2002) The curve kick of a football II: flight through the air. Sports Eng 4:193-200

de Mestre N (1990) The mathematics of projectiles in sport. Cambridge University Press, Cambridge

Grant AG, Williams AM, Reilly T (1999) Analysis of goals scored in the 1998 World Cup. J Sport Sci 17:826-827

Grealy MA, Craig CM, Bourdin C, Coleman S (2004) Judging time intervals using a model of perceptuo-motor control. J Cogn Neurosci 16:1185-1195

Lee DN, Young DS, Reddish PE, Lough S, Clayton TMH (1983) Visual timing in hitting an accelerating ball. Q J Exp Psychol A 35:333-346

Lee DN, Georgopoulos AP, Clark MJO, Craig CM, Port NL (2001) Guiding contact by coupling the taus of gaps. Exp Brain Res 139:151-159

McBeath MK (1990) The rising fastball—baseball's impossible pitch. Perception 19:545-552

McIntyre J, Zago M, Berthoz A, Lacquaniti F (2001) Does the brain model Newton's laws? Nat Neurosci 4:693-694

McLeod P, Dienes Z (1996) Do fielders know where to go to catch the ball or only how to get there? J Exp Psychol Hum Percept Perform 22:531-543

Mehta RD (1985) Aerodynamics of sports balls. Annu Rev Fluid Mech 17:151-189

Montagne G, Laurent M, Durey A, Bootsma RJ (1999) Movement reversals in ball catching. Exp Brain Res 129:87-92

Peper CE, Bootsma RJ, Mestre DR, Bakker FC (1994) Catching balls: how to get the hand to the right place at the right time. J Exp Psychol Hum Percept Perform 20:591-612

Port NL, Lee D, Dassonville P, Georgopoulos AP (1997) Manual interception of moving targets.1. Performance and movement initiation. Exp Brain Res 116:406-420

Regan D (1997) Visual factors in hitting and catching. J Sport Sci $15: 533-558$

Shanon B (1976) Aristotelianism, Newtonianism and the physics of the layman. Perception 5:241-243

Todd JT (1981) Visual information about moving objects. J Exp Psychol Hum Percept Perform 7:795-810

Werkhoven P, Snippe HP, Toet A (1992) Visual processing of optic acceleration. Vision Res 32:2313-2329

Williams M, Griffiths IW (2002) A kinematic analysis of the prevalence of pre-impact cues in the football penalty kick. J Sport Sci 20:74

Zago M, Lacquaniti F (2005) Cognitive, perceptual and actionoriented representations of falling objects. Neuropsychologia 43:178-188 